Sans résoudre l'équation, appelons $t^{\prime}$ et $t^{\prime \prime}$ les racines; nous avons $\grave{a}$ former $t^{\prime 2}+t^{\prime 2}$ et vérifier que cette somme reproduit $\frac{a^{2}}{2}$.

Or, la somme des carrés des racines d'une équation algébrique quelconque

$$
x^{m}+p x^{m-1}+q x^{m-2}+\ldots=0
$$

s'exprime par la fonction $p^{2}-2 q$, car $-p$ est la somme des racines, et $q$ la somme de leurs produits deux à deux. Ici on a

$$
p=-c \quad q=\frac{c^{2}}{2}-\frac{a^{2}}{4}
$$

et

$$
p^{2}-2 q=c^{2}-\left(c^{2}-\frac{a^{2}}{2}\right)=\frac{a^{2}}{2}
$$

ce qui justifie le résultat amnoncé.

\title{
On a Problem in Rigid Dynamics.
}

By G. M. K. IngGeTt. 